\title{
Exosomes are natural carriers of exogenous siRNA to human cells in vitro
}

\author{
Tatyana A Shtam¹', Roman A Kovalev', Elena Yu Varfolomeeva', Evgeny M Makarov², Yury V Kil ${ }^{1,3}$ \\ and Michael V Filatov ${ }^{1 *}$
}

\begin{abstract}
Background: Exosomes are nano-sized vesicles of endocytic origin that are involved in cell-to-cell communication including shuttle RNA, mainly mRNA and microRNA. As exosomes naturally carry RNA between cells, these particles might be useful in gene cancer therapy to deliver therapeutic short interfering RNA (siRNA) to the target cells. Despite the promise of RNA interference (RNAi) for use in therapy, several technical obstacles must be overcome. Exogenous siRNA is prone to degradation, has a limited ability to cross cell membranes and may induce an immune response. Naturally occurring RNA carriers, such as exosomes, might provide an untapped source of effective delivery strategies.

Results: This study demonstrates that exosomes can deliver siRNA to recipient cells in vitro. The different strategies were used to introduce siRNAs into human exosomes of various origins. The delivery of fluorescently labeled siRNA via exosomes to cells was confirmed using confocal microscopy and flow cytometry. Two different siRNAs against RAD51 and RAD52 were used to transfect into the exosomes for therapeutic delivery into target cells. The exosome-delivered siRNAs were effective at causing post-transcriptional gene silencing in recipient cells. Moreover, the exosome-delivered siRNA against RAD51 was functional and caused the massive reproductive cell death of recipient cancer cells.

Conclusions: The results strongly suggest that exosomes effectively delivered the siRNA into the target cells. The therapeutic potential of exosome-mediated siRNA delivery was demonstrated in vitro by the strong knockdown of RAD51, a prospective therapeutic target for cancer cells. The results give an additional evidence of the ability to use human exosomes as vectors in cancer therapy, including RNAi-based gene therapy.
\end{abstract}

Keywords: Exosomes, RNA interference (RNAi), Drug delivery system, Cancer therapy, RAD51

\section{Background}

The use of small interfering RNAs to induce gene silencing has opened a new avenue in drug discovery. In the past decade, efforts to develop RNA-based therapeutic technologies have been significantly intensified [1-3]. Triggering RNA interference (RNAi), in particular, has become one of the most widely used techniques for biomedical applications [4-6]. RNAi uses a mechanism of posttranscriptional sequence specific gene silencing by processing double-stranded RNAs into small-interfering RNAs (siRNAs) used as part of the RNA-induced silencing complex to selectively cleave target mRNA [7].

\footnotetext{
*Correspondence: filatov@omrb.pnpi.spb.ru

'Division of Molecular and Radiation Biophysics, SFBI Petersburg Nuclear Physics Institute, Gatchina 188300, Russia

Full list of author information is available at the end of the article
}

After the discovery that synthetic siRNAs can be exogenously introduced into cells to activate RNAi [8], this approach has become a powerful method for selective suppression of specific genes of interest in different species, showing potential for use in cancer therapeutics $[2,3]$. However, the biomedical utility of the synthetic siRNAs is limited by several RNA structure-related factors such as the negative charge (uptake by cells that also have a negatively charged surface) and instability in the blood circulation (non-modified siRNAs have a very short half-life in the blood stream, mostly because of degradation by nucleases) [9]. Another major barrier is immunogenicity of the synthetic siRNAs or delivery vehicle, especially if repeated dosing is needed to treat disease $[9,10]$. These impediments can be overcome by using natural carriers of exogenous siRNA to human 
cells. Naturally occurring RNA carriers, such as exosomes, might provide an untapped source of effective delivery strategies [11].

Exosomes are nano-sized vesicles (30-120 nm in size) produced by many cell types, including dendritic cells (DC), B cells, T cells, mast cells, epithelial cells and tumor cells [12-15]. These vesicles are formed by inward budding of late endosomes and are then released to the extracellular environment upon fusion with the plasma membrane [15]. They have been detected in body fluids such as peripheral blood, urine, malignant effusions and bronchoalveolar lavage fluid $[12,14,15]$. It has been shown that these vesicles are involved in signal transduction, antigen presentation to $\mathrm{T}$ cells and tolerance development [12,15]. In 2007, it was demonstrated that exosomes derived from various cell types contain a substantial amount of RNA (mainly mRNA and microRNA) [16]. More importantly, the exosome vesicles shuttle RNA between cells, an activity which served as a novel means of cell-to-cell communication [12,17]. The RNA present in exosomes was therefore termed exosomal shuttle RNA (esRNA) [17]. As exosomes naturally carry RNA between cells, it has been speculated that this property might be useful in gene therapy, in which a vector is used to deliver therapeutic nucleic acids to the patient's target cells [18-20]. In this report, we examined whether human exosomes can deliver exogenous nucleic acids to recipient cells in vitro.

In 2008, we investigated survivability of human cells and their ability to pass through the cell cycle after suppressing the homologous recombination genes by genespecific siRNAs [21]. We demonstrated that in most cancer cell types studied the decrease in the RAD51 protein level induced cell accumulation in S and G2 phases of the cell cycle and ultimately led to the massive reproductive cell death. Our data pointed to RAD51 as a potential target for repressing the growth of abnormally proliferating cells [21]. Here, we show that the exosome- delivered siRNA against RAD51 was also functional and caused the massive reproductive cell death of recipient cells. These findings suggest that exosomes derived from human cells can be utilized in gene cancer therapy to provide target cells with heterologous nucleic acids such as therapeutic siRNAs.

\section{Results}

Introduction of siRNA into exosomes and delivery of heterologous siRNAs to recipient cells via exosomes

Exosomes originating from HeLa and ascites were isolated by ultracentrifugation, as described earlier [22]. Size determination of isolated exosomes was performed using a Microtrac S3500 particle analyzer (Microtrac Inc) according to the manufacturer's instructions (Figure 1A). The ultracentrifuge pellets of exosomes contained spherical particles that were in accordance with the exosome size range described earlier [12,15,22]. To estimate the homogeneity of the particles the atomic force microscopy method was used. The exosomes appeared as homogeneous circular bulging vesicular structures (Figure 1B). We also confirmed the presence of exosomal marker proteins HLA-ABC and CD63 [15] on the surface of these vesicles by dot blot analysis.

Next, we investigated the possibility of loading exosomes with exogenous cargoes using chemical treatment. As chemical transfection for membrane particles at the nanometer scale is not well-characterized, nonspecific Alexa flour 488 labeled siRNA (siRNA AF488) was used for the empirical optimization of the protocol. To examine the efficiency of chemical loading of exosomes, siRNA AF488 was mixed with Lipofectamine to allow for the formation of complexes (siRNA embedded in lipid micelles), which was then added to the exosomes and incubated for $30 \mathrm{~min}$ at RT. The mixture was purified for 3-5 times by washing and ultra-filtration through a $100-\mathrm{kDa}$ filter to eliminate the excess of free siRNA as well as siRNA embedded into the micelles
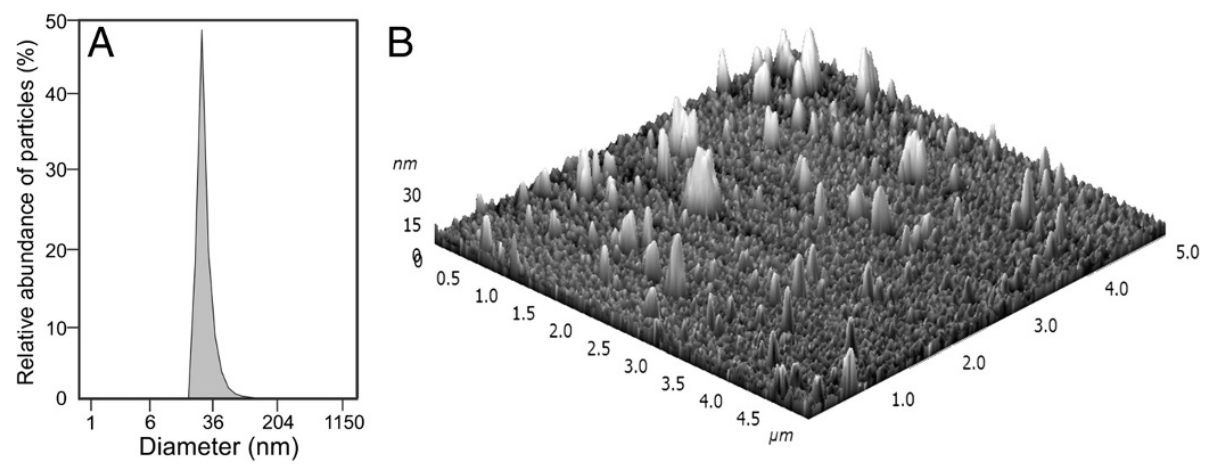

Figure 1 Characterization of exosomes originating from malighnant ascitic fluid. A, the sizes of all particles in the ultracentrifuge pellet were determined using a Microtrac \$3500 particle analyzer. B, exosomes observed under atomic force microscopy (AFM), area scan is $5 \times 5$ micrometers. 
$($ Exo + siRNA + LP + WF $)$. Exosomes loaded with siRNA were co-cultured with the recipient cells for $24 \mathrm{~h}$ in order to transfect cells with the heterologous siRNA. High delivery efficiency of siRNA by exosomes to recipient cells was confirmed by confocal microscopy and flow cytometry (Figure 2A,B). The recipient cells were also transfected with siRNA AF488 treated with Lipofectamine (siRNA + LP) in usual manner and after purifying for several times through a $100-\mathrm{kDa}$ filter as exosomal samples (siRNA + LP + WF). The results from flow cytometry and confocal microscopy showed that the fluorescence signal of the siRNA could be detected only in the cells transfected usually (siRNA + LP), as expected (Figure 2A,D). But no fluorescence signal was detected in control cells incubated with washed and ultra-filtrated transfection complexes (siRNA + LP + WF) (Figure 2A,C), confirming that the purification procedure used was sufficient to remove all nonassociated with exosomes siRNA from the samples. Thus, the results indicate the possibility of siRNA delivery into cells by exosomes in the presence of Lipofectamine. But, as siRNA embedded into the micelles could unspecifically be attached to the exosomes, it was indistinguishable whether the exosomes or the micelles of chemical transfection finally delivered exogenous nucleic acids to cells.

To examine the possibility to input siRNA into exosomes in principle we used the fluorescently labeled selfdelivering sdRNA. Self-deliverable RNA molecules do not require any transfection reagent, vehicle or special cell treatment. The sdRNA was mixed with exosomes, incubated $30 \mathrm{~min}$ at $\mathrm{RT}$ and then the exosome vesicles were purified using 3-5 times washing and ultrafiltration through a $100-\mathrm{kDa}$ filter (Amicon ultra, Millipore) to eliminate the excess of free sdRNA (Exo + sdRNA + WF). As a control the sample prepared in identical way without exosomes was used (sdRNA + WF). Then both samples were co-cultured with the recipient cells for $24 \mathrm{~h}$. The cells were also transfected with sdRNA in usual manner (sdRNA). The results from flow cytometry and confocal microscopy showed that the fluorescent signal of sdRNA was indeed associated with presence of exosomes (Figure 3A-D). SdRNA could not be detected in the cells co-cultured with control sample without exosomes, confirming that the excess of free sdRNA was depleted by purifying (Figure $3 \mathrm{C}$ ). In summary, the results strongly suggest that the approach used was successful at introducing the heterologous siRNAs into the exosomes and at delivery of the siRNAs to recipient cells via exosomes.

\section{Exosome-delivered siRNA is functional in recipient cells} Given that exosomes from HeLa and ascites could act as a carrier to deliver nonspecific heterologous genetic
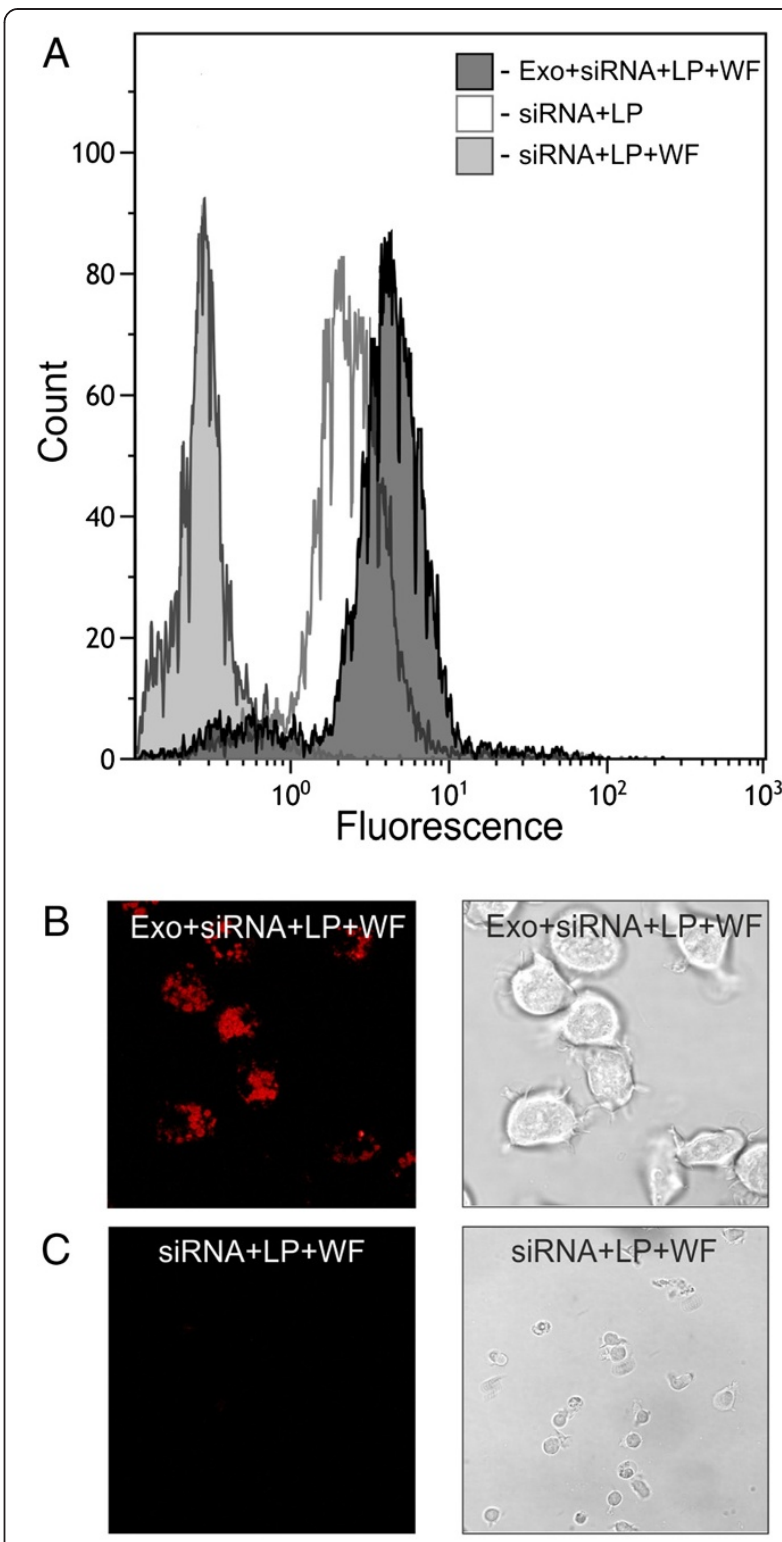

$\mathrm{D}$
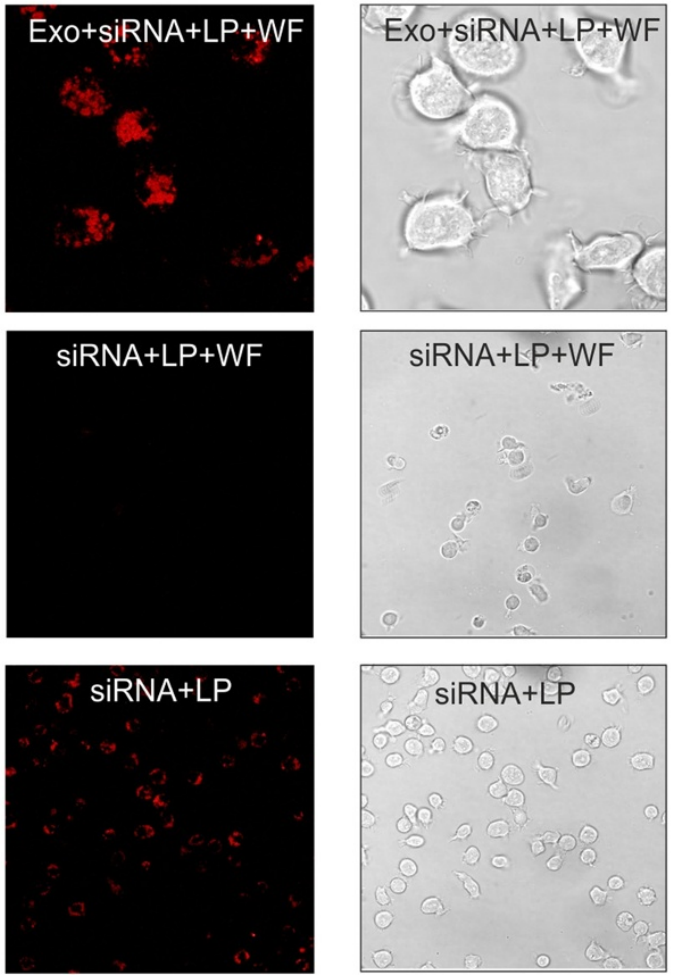

igure $\mathbf{2}$ In vitro delivery of fluorescently labeled siRNA via chemically loaded exosomes. The recipient cells were treated for $24 \mathrm{~h}$ with exosomes loaded with Lipofectamine-formulated siRNA and washed several times through a 100-kDa filter (Exo + siRNA + LP + WF). The sample prepared in identical way but without adding of exosomes was used (siRNA + LP + WF) as a control. The cells were also transfected with siRNA by standard procedure (siRNA + LP). A, flow cytometry analysis of HeLa cells transfected by siRNA. B-D, representative images of HeLa cells $24 \mathrm{~h}$ after transfection. 

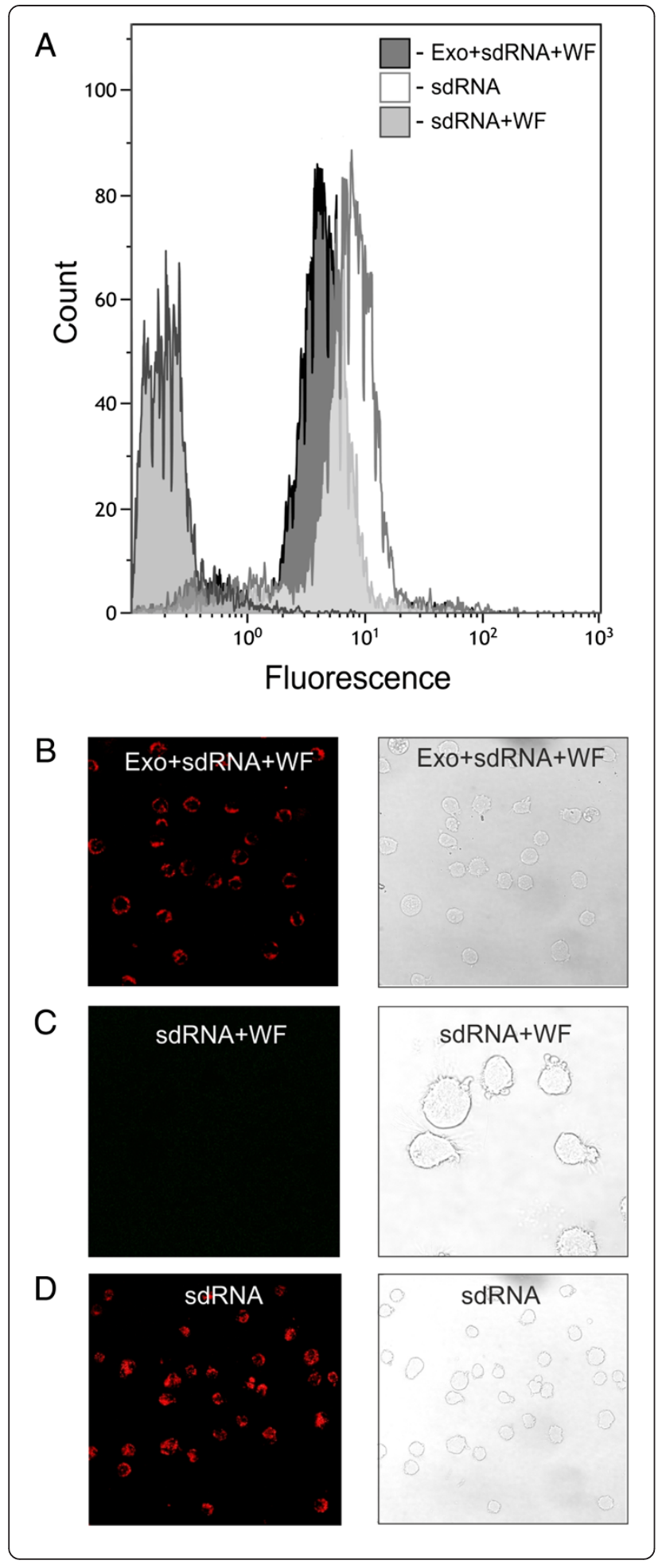

Figure 3 Transfection of HeLa cells by fluorescently labeled self-delivering sdRNA via exosomes. The recipient cells were treated for $24 \mathrm{~h}$ with exosomes loaded with the sdRNA and washed several times through a 100-kDa filter (Exo + sdRNA + WF). The sample prepared in identical way but without adding of exosomes was used (sdRNA + WF) as a control. The cells were also transfected with sdRNA by standard procedure (sdRNA). A, flow cytometry analysis of HeLa cells transfected by sdRNA. B-D, representative images of HeLa cells $24 \mathrm{~h}$ after transfection.

material to recipient cells, we investigated whether the specific siRNAs delivered via exosomes to cells would down-regulate the expression of target genes and, if so, whether the exosome-delivered siRNA would be functional in recipient cells. Therefore, we targeted RAD51 and RAD52 genes by specific siRNAs delivered via exosomes to HeLa and HT1080 cells to evaluate the therapeutic potential of this technology. The RAD51 recombinase executes the central functions in homologous recombination: the search for a homologous template DNA and the formation of the joint heteroduplex molecule between the damaged DNA and the undamaged template [23]. In addition to RAD51, homologous recombination requires the coordinated action of a number of other proteins of homologous recombination, including RAD52, which can bind DNA ends and anneal complementary singlestranded DNA molecules [24]. The role of homologous recombination in the maintenance of stable genome and viability of somatic mammalian cells is still under investigation. We have shown previously that depression of the RAD51 gene function leads to the massive reproductive death of human cancer cells in the absence of genotoxic injuries [21]. Our data demonstrated that the significant down-regulation of RAD51 but not RAD52 protein by the specific siRNA resulted in S/G2 cell cycle blocks. In most of the cancer cell lines such blocks resulted in dramatic decrease in cell viability accompanied by apoptosis or irreversible loss of their ability to proliferate. Therefore, we pointed to RAD51 as a potential target to depress the abnormally proliferating cells [21]. Here RAD51 and RAD52 were knockdown by specific siRNAs in HeLa and HT1080 cells via genotoxic delivery by exosomes derived from $\mathrm{HeLa}$ and ascitic fluids. Both cell lines were co-cultured with exosomes chemically loaded with the RAD51 or RAD52 siRNA for 72-96 h. Western blot analysis showed a considerable reduction in both RAD51 and RAD52 protein levels in cells as after transfection with specific siRNAs via exosomes and after siRNA standard cell transfection with Lipofectamine (Additional file 1: Figure S1A). Moreover the recruitment of RAD51 at double-strand breaks induced in HeLa cells by ionizing radiation was reduced in cells treated by exosomes loaded with antiRAD51 siRNA (Additional file 1: Figure S1B). 


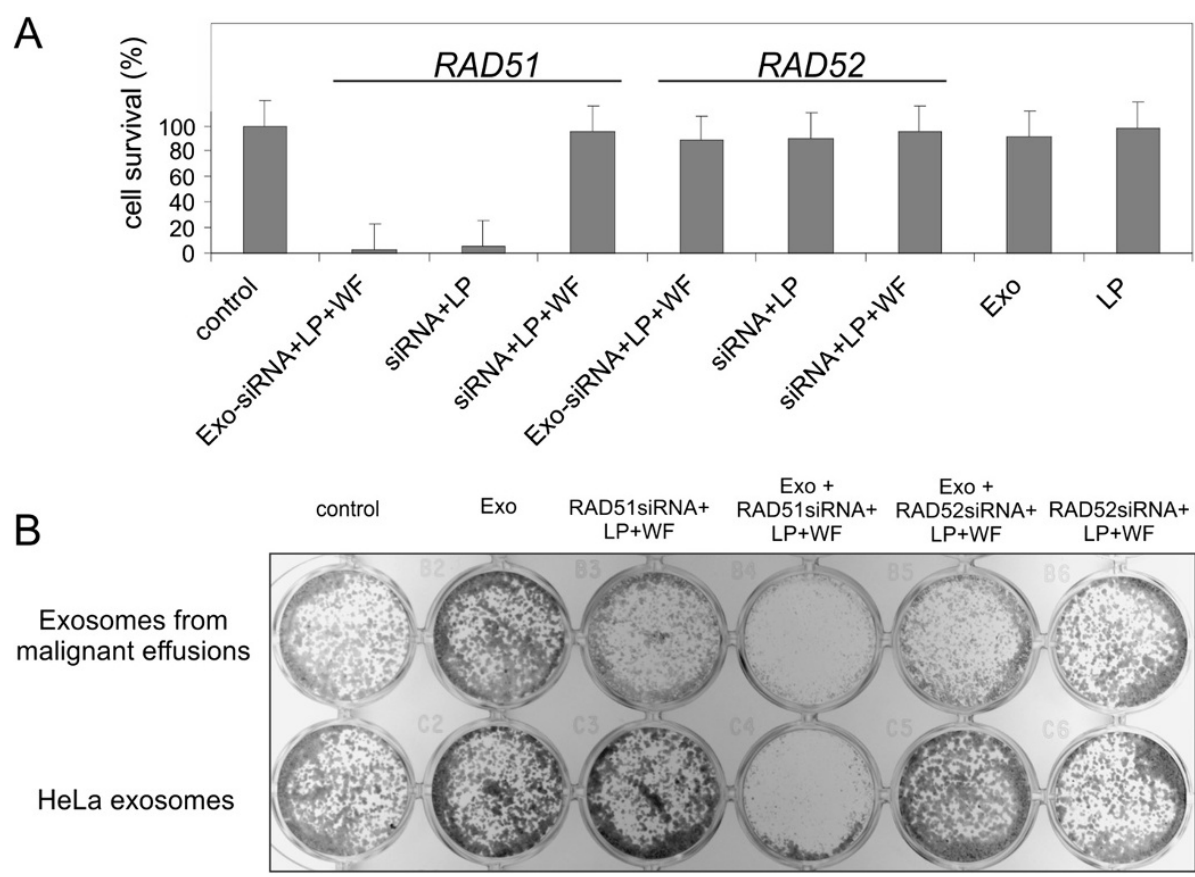

Figure 4 RAD51 down-regulation by exosome delivered siRNA leads to dramatic decrease of viability of human cells. A, the survival of HeLa cells after RAD51 and RAD52 depletion by two ways: by direct specific siRNA chemical transfection (siRNA + LP) and by chemically loaded with siRNA exosomes (EXo + siRNA + LP + WF). The survival of the non-transfected cells was assumed as 100\% (control). Error bars represent the standard error of the mean (SE) of at least three independent experiments. B, the example of clonogenic cell survival of the HeLa cells after co-culturing with ascites-derived exosomes (upper row) or HeLa exosomes (bottom row) which act as a carrier to deliver specific siRNAs to recipient cells.

Next we examined the colony forming ability of RAD51 or RAD52 knocked down cells. The total amount of colonies was counted in 5-7 days after siRNA was added (Figure 4). Standard transfection with siRNAantiRAD51 resulted in a significant decrease of HeLa and HT1080 cell survival (siRNA + LP). At the same time transfection with siRNA-antiRAD52 had no effect on the cell survival. The same results were observed for the cells transfected by the siRNAs via exosomes (Exo + siRNA + LP + WF). Neither Lipofectamine alone (LP) nor exosomes alone (Exo) nor purified by washing and filtering siRNAs samples (siRNA + LP + WF) had significant effect on the cell survival.

We also examined the influence of the RAD51 protein on the cell cycle parameters. As shown in Figure 5A, silencing of the RAD51 gene via the siRNA loaded exosomes during 24-48 $\mathrm{h}$ induced the accumulation of S-phase and G2-phase cells. More prolonged RAD51 siRNA transfection via exosomes $(72 \mathrm{~h})$ caused the block of the recipient cells mainly in the G2/M phase and apoptotic cells death, which was indicated by high degree of cell DNA degradation (Figure 5A). Apoptosis was also studied by flow cytometry with mitochondrial membrane potential and membrane integrity fluorochromes. Both DiOC6(3)/PI and Yo-Pro-1/PI double staining flow cytometry showed that the apoptotic rate of HeLa cells transfected by RAD51 siRNA via exosomes was increased compared with that in the control cells (Figure 5B,C). In addition, cell morphology was determined in RAD51 depleted cells. Figure 5D shows the morphology of HeLa cells stained with Hoechst 33342. The confocal images showed that control cells possessed intact nuclei. The RAD51 siRNA transfection via exosomes caused the formation of degraded nuclei, membrane blebbing and clear apoptotic bodies (Figure 5D).

Thus, exosomes from HeLa and ascites were able to deliver heterologous siRNAs to human cells. Importantly, the exosome-delivered RAD51 siRNA was functional and caused post-transcriptional gene silencing, induced accumulation of the cells in $\mathrm{S}$ and G2/M phases of cell cycle and resulted in recipient cell death. Interestingly, the exosomes from different ascites were able to deliver heterologous siRNAs to human cultured cells such as HeLa or HT1080 cells (Figure 4B). This observation indicates the nonspecific ability of the cells to capture alien vesicles.

As mentioned above, we could not exclude adhesion of siRNA embedded into the micelles on the surface of exosomes in the presence of Lipofectamine. However, the location of siRNA outside of exosomes would lead to problems in delivery of safe siRNA in vivo. Therefore, electroporation was examined as a means of introducing 


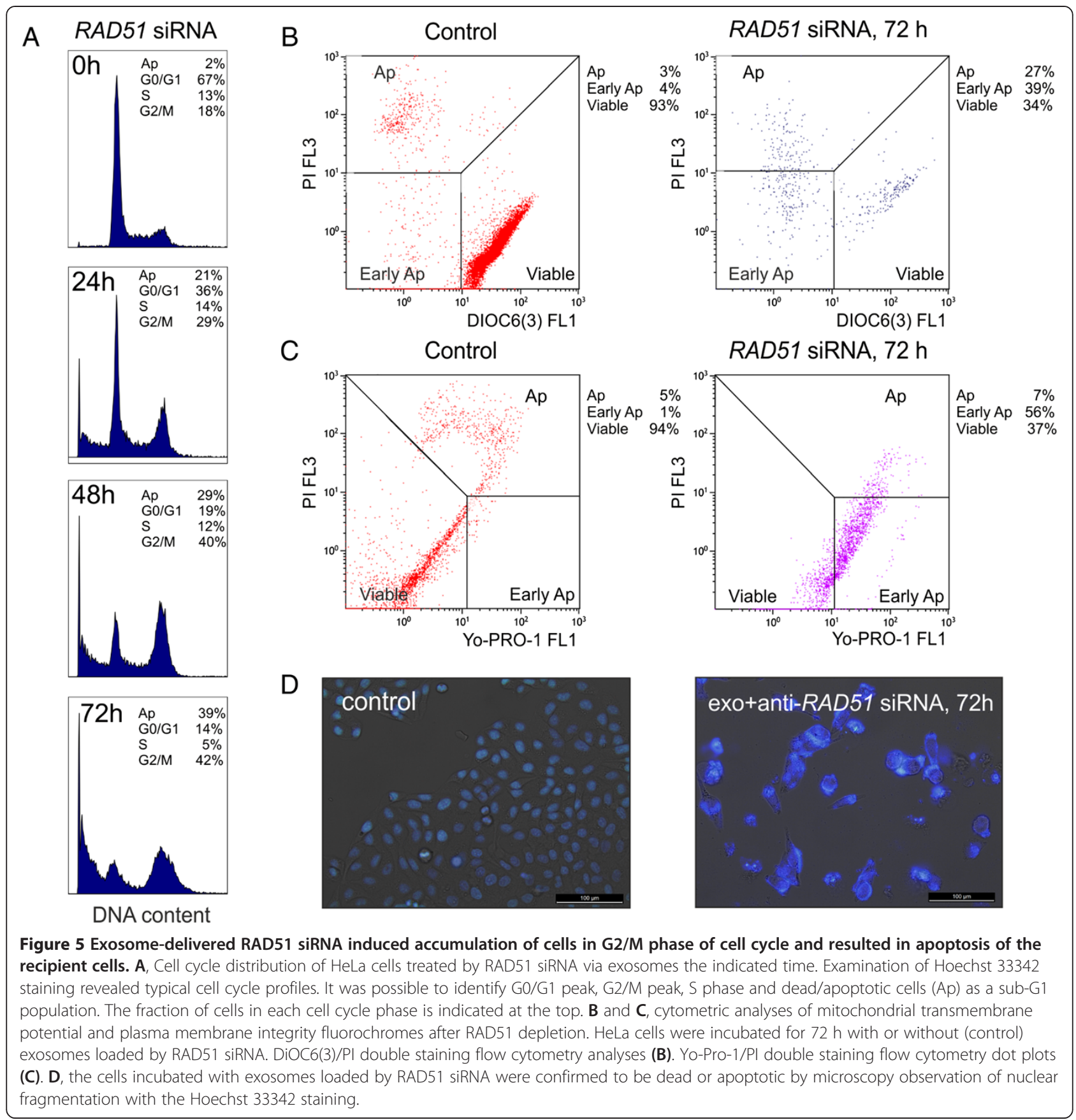

genetic material into exosomes. Having discovered the optimal parameters for transferring siRNA into exosomes by electroporation, siRNA against RAD51 was packaged into exosomes derived from HeLa cells. Survival of the cells after co-culturing with electroporated exosomes was used as a test system to optimize the transfection efficiency. The results shown in Figure 6 provide evidence that the heterologous siRNA was introduced into exosomes by electroporation. Summing up the data, we can assume that exosomes effectively delivered the siRNA into the target cells, causing selective genes silencing and leading to reproductive cancer cell death by knockdown of RAD51.

\section{Discussion}

The identification of extracellular phospholipid vesicles as conveyors of cellular information has created excitement in the field of drug delivery [12]. Biological therapeutics, including short interfering RNA are prone to degradation, have limited ability to cross biological membranes, and 


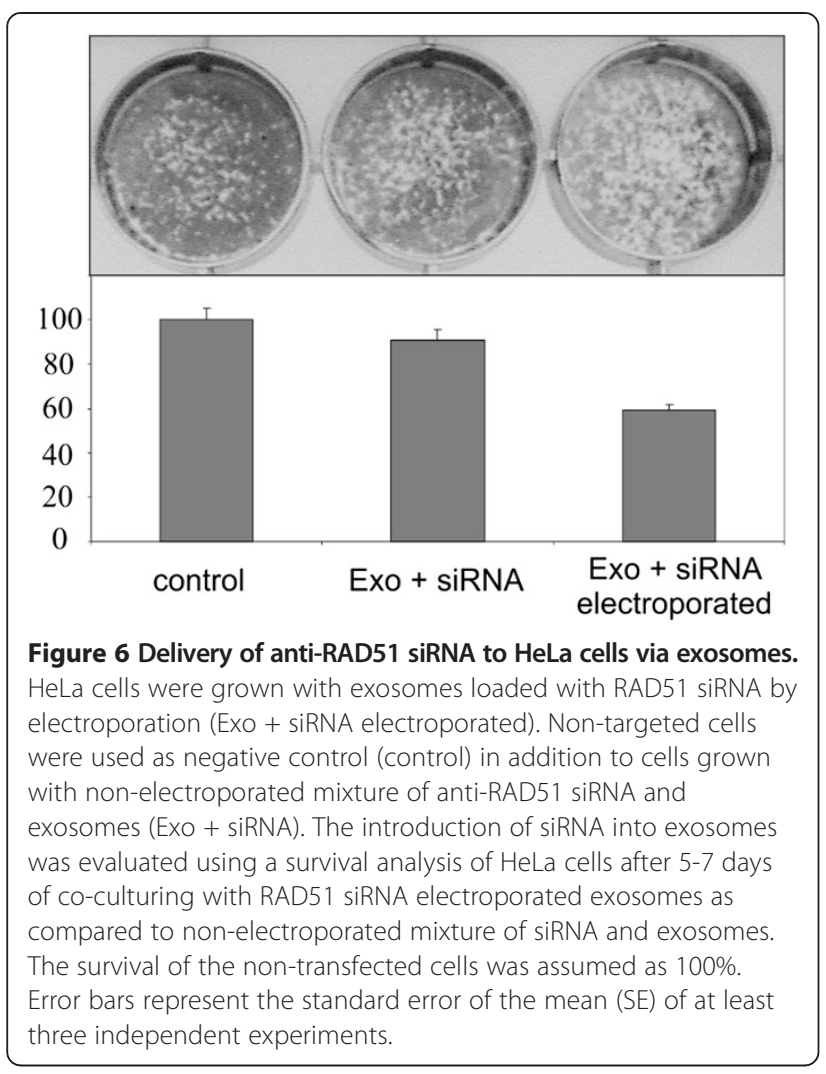

may elicit immune responses [3]. Therefore, delivery systems for such drugs are under intensive investigation [25]. Exploiting extracellular vesicles as carriers for biological therapeutics is a promising strategy to overcome these issues and to achieve efficient delivery to the cytosol of target cells [19]. Exosomes are a well-studied class of extracellular vesicles known to carry proteins and nucleic acids, making them especially suitable for such strategies. Exosomes and microvesicles are naturally adapted for the transport and intracellular delivery of proteins and nucleic acids. This makes them particularly attractive for the delivery of pharmaceutical proteins and nucleic acids, such as siRNA $[19,25]$. Intracellular delivery of siRNA is a challenging task, given that naked siRNAs are rapidly degraded in the circulation, their size and negative charge limits membrane passage and cellular uptake, some siRNA sequence motifs may elicit undesired immune responses, and targeting to specific tissues and cells is required to reduce adverse effects caused by off-target silencing $[26,27]$. Encapsulation of nucleic acid-based therapeutics in endogenous transporting vesicles is a promising novel strategy to overcome most of these delivery issues. Exosomes may be most suitable for such strategies, because they are small $(30-120 \mathrm{~nm})$, relatively homogenous in size, and well studied. Their size is advantageous for their use as drug delivery systems, because this allows them to evade rapid clearance by the mononuclear phagocyte system and enhances passage through fenestrations in the vessel wall, as might occur during inflammation [28].

The successful use of exosomes for the targeted delivery of siRNA has been recently demonstrated by AlvarezErviti et al. [29]. They harvested dendritic cells from mice and transfected them to express the neuronal targeting ligand, RVG, coupled to the exosomal membrane protein, Lamp2b. This protein was expressed by the cells and incorporated in secreted exosomes. The exosomes were harvested, purified, and loaded with siRNA against an important protein in Alzheimer pathogenesis (BACE1) by electroporation. When the modified exosomes were injected intravenously in wild-type mice, a $60 \%$ decrease of BACE1 mRNA in the brain cortex was observed after 3 days. Moreover, no increase in serum interleukin-6, interferon gamma-induced protein 10, tumor necrosis factor alpha and interferon alpha concentrations was observed after injection of the exosomes, suggesting that the modified exosomes were immunologically inert. The biotechnological approach to create exosome-based delivery systems used by Alvarez-Erviti et al. was the first demonstration of an exosome-based drug delivery system which showed efficient in vivo delivery of siRNA [29]. In 2012, Wahlgren et al described an "exosome display technology in vitro" in which, exogenous siRNAs were successfully introduced into various kinds of human exosomes and were used to deliver siRNA to human mononuclear blood cells. Plasma exosomes effectively delivered the siRNA into the target cells, causing selective gene silencing of MAPK-1 [30]. Other strategies to exploit exosomes for siRNA delivery in vitro and in vivo have also been reported [25,31,32]. But, despite promising uses for exosomes as a delivery of exogenous RNA, to date, only a few research reports on the subject were published.

The purpose of this study was to investigate the potential use of exosomes as a carrier for siRNA delivery in vitro. Fluorescently labeled siRNAs were successfully introduced into various kinds of human exosomes: conventional siRNA was loaded with a chemical transfection reagent (Lipofectamine) and self-delivering sdRNA without any reagent. Both cultural and ascites-derived exosomes effectively delivered the hydrophobically modified sdRNA or usual siRNA molecules into the different target cells. This observation indicates the nonspecific ability of the cells to capture alien vesicles. Taken together, the results strongly suggest that the approaches used were successful at introducing the heterologous siRNAs into the exosomes and at delivery of heterologous siRNAs to recipient cells via exosomes.

Next, we examined if exosome-delivered siRNA was functional and caused post-transcriptional gene silencing in recipient cells. The RAD51 gene was found to be down-regulated in HeLa and HT1080 cells that had been co-cultured with exosomes containing siRNA against the 
RAD51 transcript. The results show that exosomes effectively delivered the siRNA against RAD51 into the target cells, causing selective genes silencing and leading to reproductive cancer cell death by knockdown of RAD51. The effect of RAD51 gene knockdown was equally visible in HeLa and in HT1080 cells. This indicates approximately the same ability of these cancer cell types to adsorb exosomes. Our results, and the results published previously [30], show that chemical loading of the exosomes to be used as siRNA delivery vector was inapplicable as the excess of micelles (siRNA embedded in lipid micelles) could not be separated from the exosomes. It is uncertain whether the exosomes or the excess of micelles deliver the nucleic acid of interest to cells and, therefore, safe delivery of siRNA via exosomes is not evident in the long term use this approach in vivo. Thus, electroporation was examined as a means of introducing siRNA against RAD51 into exosomes. Survival of HeLa cells after co-culturing with electroporated exosomes was used as a test system to optimize the transfection. The results provide sufficient evidence that the heterologous siRNA was quite effectively introduced into exosomes using electroporation, but the method may need to be optimized for each exosome and each cell type.

\section{Conclusions}

We can assume that exosomes effectively deliver siRNA into the target cells in vitro, causing selective genes silencing and leading to reproductive cancer cell death by knockdown of RAD51 recombinase. Taken together, (i) exosomes may represent an efficient delivery platform for siRNAs, (ii) siRNA-mediated induction of RNAi is a promising approach for the knockdown of pathologically relevant oncogenes, and (iii) delivery of siRNA via exosomes may become an attractive therapeutic strategy for the treatment of cancer.

\section{Methods}

\section{Exosome purification}

For the generation of exosomes HeLa and HT1080 human fibosarcoma cells were grown during 5 days in $30 \mathrm{ml}$ $\left(250 \mathrm{sm}^{2}\right.$ flacks were used) of DMEM-F12 medium supplemented with $10 \%$ fetal bovine serum (FBS) depleted from exosomes as described [22]. On the day of collection, the medium from the cells was removed (conditioned medium). Exosomes were isolated by differential centrifugations and micro-filtration as previously described $[15,22]$. Briefly, $100 \mathrm{ml}$ of cell supernatants were harvested, centrifuged at $300 \mathrm{~g}$ for $10 \mathrm{~min}$ to eliminate cells and then at $15000 \mathrm{~g}$ for $30 \mathrm{~min}$ to remove cell debris. Exosomes were pelleted by ultracentrifugation at 110 $000 \mathrm{~g}$ for $70 \mathrm{~min}$ at $4^{\circ} \mathrm{C}$. The exosome pellet was resuspended in $1 \mathrm{ml}$ of DMEM medium by syringing three to five times through a sterile 27-gauge needle to prevent the exosomes from clumping together. Then the exosome samples were filtered through a $0.22-\mu \mathrm{m}$ filter for sterilization. The protein content of the exosome suspension was quantified using the Bradford reagent. Exosomes from malighnant ascitic fluids (ascites) were prepared by differential centrifugation and filtration as for HeLa and HT1080 cells.

\section{Atomic force microscopy (AFM)}

Purified exosomes were diluted in de-ionized water and adsorbed to freshly cleaved mica sheets, fixed with $0.5 \%$ glutaraldehyde, rinsed with de-ionized water and airdried. The samples were scanned in the air by semicontact method with a scanning microscope of Solver Bio series (NT-MDT, Russia) equipped with silicon test probe (type NSG01), with a characteristic stiffness of $5.5 \mathrm{~N} / \mathrm{m}$ and a typical radius of curvature of the tip (less than $10 \mathrm{~nm}$ ). The initial amplitude of scanning was set to $16 \mathrm{nA}$ in current terms; SetPoint was adjusted to half of the amplitude. Scanning was performed with a frequency of $1.01 \mathrm{~Hz}$. The images were processed using standard software package (Image Analysis Nova).

\section{Loading of exosomes with siRNA}

Two different methods, chemical treatment and electroporation, were used. For chemical loading of exosomes, siRNA at a final concentration of $2 \mu \mathrm{mol} / \mathrm{ml}$ was mixed with Lipofectamine transfection reagent in $100 \mu \mathrm{l}$ of siRNA buffer and incubated for $10 \mathrm{~min}$ at RT. Then $300 \mu \mathrm{l}$ of exosome suspension was added and the mixture was incubated for additional $30 \mathrm{~min}$ at RT. To remove the excess of micelles, the exosomes were purified using three to five times filtration through a $100-\mathrm{kDa}$ filter (Amicon, Millipore) with washing by cultural medium.

For electroporation assays exosomes derived from HeLa, HT1080 or ascitic fluids were diluted in cytomix transfection buffer. SiRNAs against RAD51 or RAD52 at a final concentration of $2 \mu \mathrm{mol} / \mathrm{ml}$ were added to $300 \mu \mathrm{l}$ of exosome sample. The mixtures were transferred into ice cold $0.4-\mathrm{cm}$ cuvettes and electroporated at $0.7 \mathrm{kV}$ using 350 microsecond pulse 20 times by the Eppendorf multiporator.

RNA duplexes targeting RAD51 or RAD52 (ON-TARGETplus SMARTpool) were purchased from Dharmacon. For control experiments fluorescently labeled conventional siRNA (Qiagen) and self-delivering siRNA (sdRNA) were used. SdRNA, a hydrophobically modified siRNA for cell transfection without formulation with Lipofectamine, was kindly provided by Advirna, Cambridge MA.

\section{Transfection of siRNA into cells via exosomes}

For siRNAs exosome-delivery assays, HeLa and HT1080 cells were seeded in 24-well plates at a density of $0.5 \times$ 
$10^{4}$ cells/well. A $30 \mu \mathrm{l}$ of purified samples, containing exosomes loaded with siRNA were co-cultured with the recipient cells in order to deliver the heterologous siRNA. The cells, transfected with a fluorescently labeled siRNA, were trypsinized, then isolated, washed three times with PBS and were analyzed by Confocal microscopy (LEICA TCS SP5X) and Flow cytometry (Beckman Coulter). All tissue culture reagents were obtained from Invitrogen and Hyclone.

\section{Protein detection}

Dot blot was used to detect exosomal proteins HLA-ABC and CD63 in exosome suspension after purification. Fifteen micrograms of exosome suspension were dropped into nitrocellulose (NC) membranes (Millipore). The membranes were dried, blocked with $5 \%$ nonfat dry milk, washed in Tris-Buffered Saline with $0.1 \%$ Tween 20 buffer (TBST) and probed with primary antibodies against HLAABC (1:200 dilution) or CD63 (1:200 dilution). All the primary antibodies were obtained from Beckman Coulter. After washing in TBST, membranes were incubated with HRP-conjugated secondary antibody (antimouse IgG: 1:10000; Sigma). For detection, enhanced chemiluminesence was carried out using the ECL plus kit (Amersham Biosciences Corp).

The exosome-based delivery of siRNA to cells was determined by Western Blot analysis and compared with direct transfection of the same siRNA to cells. For expression analysis of RAD51 and RAD52 proteins whole cell extracts were made by lysis of $1-5 \times 10^{6}$ cells in 30-50 $\mu \mathrm{l}$ of lysis buffer (10 $\mathrm{MM}$ Tris- $\mathrm{HCl} \mathrm{pH} \mathrm{7.4,0.1 \%} \mathrm{Triton}$ $\mathrm{X}-100,5 \mathrm{mM} \mathrm{PMSF}, 5 \mathrm{mM} \mathrm{MgCl}_{2}, 5 \mathrm{u} / \mathrm{ml}$ DNAse I, 20 мM $\beta$-mercaptoethanol). For western analysis aliquots of extracts containing equal amounts of total protein as determined by Bradford reagent were used. Following sonication and boiling, aliquots were resolved by $12 \%$ SDS-PAGE according to Laemmli, transferred to a PVDF membrane (Thermo Scientific) and hybridized with a mouse monoclonal antibody against RAD51 at the 1:1000 dilution (clone 3C10, Invitrogen) or against RAD52 at the 1:1000 dilution (Thermo Scientific) followed by a peroxidase-labeled anti-mouse antibody (Sigma) at the 1:10000 dilution. Antibody binding was detected by enhanced chemoluminescence (Pierce). Equality of loading was confirmed by hybridizing with a monoclonal antibody against GAPDH at the 1:10000 dilution (Clone: ZG003, Zymed).

\section{Clonogenic survival assays}

After treatment with exosomes loaded with siRNAs as stated above the cells were cultured for several days. After 5-7 days the plates were rinsed with PBS, stained with methylene blue $(0.25 \%)$ and visible colonies were counted. The survival of the non-transfected cells was assumed as $100 \%$.

\section{Cell cycle analysis}

For monitoring of cell cycle parameters $30-40 \%$ confluent HeLa cells, seeded in a $35 \mathrm{~mm}$ diameter Petri dishes, were incubated with exosomes loaded with RAD51 siRNA for 24-72 hours. After incubation, cells were trypsinized, centrifuged at $200 \mathrm{~g}$ for $10 \mathrm{~min}$, washed with PBS and then stained with a dye solution $(1 \mu \mathrm{g} / \mathrm{ml}$ Hoechst 33342, $0.1 \%$ Triton X-100) for 10 min and subjected to flow cytometry analysis. Except for RAD51 siRNA, the control cells were subjected to the same experimental conditions.

\section{Apoptosis assay}

DiOC6(3)/PI staining was used to detect mitochondrial membrane potential assessment and plasma membrane integrity. A total of $10^{6}$ cells were diluted in $100 \mu \mathrm{l}$ of PBS. DiOC6(3) (Invitrogen) was added up to a final concentration of $20 \mathrm{nM}$. The tubes were then gently mixed and incubated for $20 \mathrm{~min}$ in $5 \% \mathrm{CO}_{2}$ atmosphere at $37^{\circ} \mathrm{C}$. Then the cells were washed with PBS, contained $2 \%$ of serum, and PI (Propidium Iodide) was added up to a final concentration of $1 \mu \mathrm{g} / \mathrm{ml}$. Flow cytometry analysis was conducted within $10 \mathrm{~min}$. The data from $10^{4}$ cells were collected and analyzed using CellQuest Pro Software (Becton Dickinson) to calculate the proportion of early apoptotic, late apoptotic/dead and viable healthy cells.

The Vybrant Apoptosis Assay Kit (Molecular Probes) was used to detect changes in plasma membrane permeability to Yo-Pro-1 related to apoptosis. A total of $10^{6}$ cells were diluted in $1 \mathrm{ml}$ of PBS; $1 \mu \mathrm{l}$ of Yo-Pro- $1(100 \mu \mathrm{mol} / \mathrm{l})$ was added. The tubes were gently mixed and incubated for $20 \mathrm{~min}$ at RT and $6 \mu \mathrm{mol} / \mathrm{l}$ PI were added to each tube. Flow cytometry analysis was conducted within $10 \mathrm{~min}$.

\section{Immunofluorescence analysis of RAD51 foci}

Before irradiation HeLa cells were incubated for $48 \mathrm{~h}$ with exosomes loaded with RAD51siRNA. Gamma irradiation was performed with a ${ }^{137} \mathrm{Cs}$ source. After irradiation at 10 Gy the cells were maintained for $6 \mathrm{~h}$ in the fresh medium and then analyzed by immunofluorescence for the presence of RAD51 foci. Immunofluorescence staining was performed by $4 \%$ PFA fixation, $0.2 \%$ Triton X-100 treatment, blocking in $1 \%$ BSA plus $1 \%$ normal goat serum and staining with first mouse monoclonal antibody against RAD51 at the 1:200 dilution (clone 3C10, Invitrogen) and Alexa Fluor 594 secondary antibodies (Invitrogen). Except for RAD51 siRNA, the control cells were subjected to the same experimental conditions.

\section{Endnotes}

This work was performed with financial assistance of Russian Federal Program "Scientific and ScientificPedagogical Personnel of Innovative Russia", contract 14.740.11.0754 and Fellowship from the Administration of Leningrad region to Shtam T. 


\section{Additional file}

Additional file 1: Figure S1. siRNA transfection via exosomes resulted in a substantial decrease of the protein expression level and in suppression of RAD51 downstream activity. A, Western blot probed with RAD51 and RAD52 in HeLa cells direct transfected with specific siRNAs and with exosome carriers of siRNAs against RAD51 or RAD52. The cells treated by Lipofectamine alone were analyzed as a control. Equality of loading was confirmed by hybridizing with a monoclonal antibody against GAPDH. B, Analysis of RAD51 recruitment in HeLa cells irradiated with $\gamma$-rays. Representative pictures of RAD51 repair foci in HeLa cells at $6 \mathrm{~h}$ after irradiation with $10 \mathrm{~Gy}$, control (left panel) and cells transfected by RAD51 siRNA via exosomes (right panel).

\section{Abbreviations}

RNAi: RNA interference; siRNAs: Small-interfering RNAs; LP: Lipofectamine; AFM: Atomic force microscopy; PI: Propidium iodide.

\section{Competing interests}

The authors declare that they have no competing interests.

\section{Authors' contributions}

MVF and TAS conceived and designed the experiments. TAS, RAK and EYV carried out the experiments. EMM contributed into analysis and interpretation of data. TAS, YVK and MVF wrote the manuscript. All authors read and approved the final manuscript.

\section{Acknowledgments}

We thank Dr. Ludmila Molodkina of the St. Petersburg State Polytechnical University for advice and technical assistance in size determination of exosomes by Microtrac S3500 particle analyzer.

\section{Author details}

${ }^{1}$ Division of Molecular and Radiation Biophysics, SFBI Petersburg Nuclear Physics Institute, Gatchina 188300, Russia. ${ }^{2}$ School of Health Sciences and Social Care, Brunel University, Uxbridge UB8 3PH, UK. ${ }^{3}$ Department of Biophysics, St. Petersburg State Polytechnical University, St. Petersburg 195251, Russia.

Received: 24 July 2013 Accepted: 29 October 2013

Published: 18 November 2013

\section{References}

1. Davis ME: The first targeted delivery of siRNA in humans via a self-assembling, cyclodextrin polymer-based nanoparticle: from concept to clinic. Mol Pharm 2009, 6:659-668.

2. Davis ME, Zuckerman JE, Choi CH, Seligson D, Tolcher A, Alabi CA, Yen Y, Heidel JD, Ribas A: Evidence of RNAi in humans from systemically administered siRNA via targeted nanoparticles. Nature 2010, 464:1067-1070.

3. Pecot CV, Calin GA, Coleman RL, Lopez-Berestein G, Sood AK: RNA interference in the clinic: challenges and future directions. Nat Rev Cancer 2011, 11:59-67.

4. Kole R, Krainer AR, Altman S: RNA therapeutics: beyond RNA interference and antisense oligonucleotides. Nat Rev Drug Discov 2012, 11:125-140.

5. Guo P: The emerging field of RNA nanotechnology. Nat Nanotechnol 2010, 5:833-842.

6. Bramsen JB, Kjems J: Development of therapeutic-grade small interfering RNAs by chemical engineering. Front Genet 2012, 3:154.

7. Fire A, Xu S, Montgomery MK, Kostas SA, Driver SE, Mello CC: Potent and specific genetic interference by double-stranded RNA in Caenorhabditis elegans. Nature 1998, 391:806-811.

8. Elbashir SM, Lendeckel W, Tuschl T: RNA interference is mediated by 21- and 22-nucleotide RNAs. Genes Dev 2001, 15:188-200.

9. Seow Y, Wood MJ: Biological gene delivery vehicles: beyond viral vectors. Mol Ther 2009, 17:767-777.

10. Foged C: SiRNA delivery with lipid-based systems: promises and pitfalls. Curr Top Med Chem 2012, 12:97-107.

11. El-Andaloussi S, Mäger I, Breakefield XO, Wood MJ: Extracellular vesicles: biology and emerging therapeutic opportunities. Nat Rev Drug Discov 2013, 12:347-357.
12. Vlassov AV, Magdaleno S, Setterquist R, Conrad R: Exosomes: current knowledge of their composition, biological functions, and diagnostic and therapeutic potentials. Biochim Biophys Acta 2012, 1820:940-948.

13. Tan A, Rajadas J, Seifalian AM: Exosomes as nano-theranostic delivery platforms for gene therapy. Adv Drug Deliv Rev 2013, 65:357-367.

14. Azmi AS, Bao B, Sarkar FH: Exosomes in cancer development, metastasis, and drug resistance: a comprehensive review. Cancer Metastasis Rev 2013, 1:20. Doi: 10.1007/s10555-013-9441-9.

15. Mathivanan S, Ji H, Simpson RJ: Exosomes: Extracellular organelles important in intercellular communication. J Proteomics 2010, 73:1907-1920

16. Valadi H, Ekström K, Bossios A, Sjöstrand M, Lee JJ, Lötvall JO: Exosome-mediated transfer of mRNAs and microRNAs is a novel mechanism of genetic exchange between cells. Nat Cell Biol 2007, 9:654-659.

17. Eldh $M$, Ekström $K$, Valadi $H$, Sjöstrand $M$, Olsson B, Jernås $M$, Lötvall J: Exosomes communicate protective messages during oxidative stress; possible role of exosomal shuttle RNA. PLoS One 2010, 5:e15353.

18. Lässer C, Alikhani VS, Ekström K, Eldh M, Paredes PT, Bossios A, Sjöstrand M, Gabrielsson S, Lötvall J, Valadi H: Human saliva, plasma and breast milk exosomes contain RNA: uptake by macrophages. J Transl Med 2011, 9:9.

19. O'Loughlin AJ, Woffindale CA, Wood MJ: Exosomes and the emerging field of exosome-based gene therapy. Curr Gene Ther 2012, 12:262-274.

20. György B, Szabó TG, Pásztói M, Pál Z, Misják P, Aradi B, László V, Pállinger E, Pap E, Kittel A, et al: Membrane vesicles, current state-of-the-art: emerging role of extracellular vesicles. Cell Mol Life Sci 2011, 68:2667-2688.

21. Shtam TA, Varfolomeeva EY, Semenova EV, Filatov MV: Role of human RAD51 recombinase in the cycle checkpoint and survival of a cell. Cell Tissue Biol 2008, 2:463-467.

22. Simpson RJ, Jensen SS, Lim JW: Proteomic profiling of exosomes: current perspectives. Proteomics 2008, 8:4083-4099.

23. Baumann P, Benson FE, West SC: Human Rad51 protein promotes ATP-dependent homologous pairing and strand transfer reactions in vitro. Cell 1996, 87:757-766.

24. Liu Y, Maizels N: Coordinated response of mammalian Rad51 and Rad52 to DNA damage. EMBO Rep 2000, 1:85-90.

25. Kooijmans SA, Vader P, Van Dommelen SM, Van Solinge WW, Schiffelers RM: Exosome mimetics: a novel class of drug delivery systems. Int J Nanomedicine 2012, 7:1525-1541.

26. Castanotto D, Rossi JJ: The promises and pitfalls of RNA-interference based therapeutics. Nature 2009, 457:426-433.

27. Baigude H, Rana TM: Delivery of therapeutic RNAi by nanovehicles. ChemBioChem 2009, 10:2449-2454.

28. van den Boorn JG, Schlee M, Coch C, Hartmann G: SiRNA delivery with exosome nanoparticles. Nat Biotechnol 2011, 29:325-326.

29. Alvarez-Erviti L, Seow Y, Yin H, Betts C, Lakhal S, Wood MJ: Delivery of siRNA to the mouse brain by systemic injection of targeted exosomes. Nat Biotechnol 2011, 29:341-345.

30. Wahlgren J, Karlson TDL, Brisslert M, Sani FV, Telemo E, Sunnerhagen P, Valadi H: Plasma exosomes can deliver exogenous short interfering RNA to monocytes and lymphocytes. Nucleic Acids Res 2012, 40:e130.

31. El-Andaloussi S, Lee Y, Lakhal-Littleton S, Li J, Seow Y, Gardiner C, Alvarez-Erviti L, Sargent IL, Wood MJA: Exosome-mediated delivery of siRNA in vitro and in vivo. Nat Protoc 2012, 7:2112-2126.

32. Koppers-Lalic D, Hogenboom MM, Middeldorp JM, Pegtel DM: Virus-modified exosomes for targeted RNA delivery; A new approach in nanomedicine. Adv Drug Deliv Rev 2013, 65:348-356.

\section{doi:10.1186/1478-811X-11-88}

Cite this article as: Shtam et al:: Exosomes are natural carriers of exogenous siRNA to human cells in vitro. Cell Communication and Signaling 2013 11:88 